\title{
Impairment of neutrophilic glucocorticoid receptor function in patients treated with steroids for septic shock
}

\author{
Maria Bergquist ${ }^{1,2^{*}}$, Catharina Lindholm², Morten Strinnholm ${ }^{3}$, Göran Hedenstierna ${ }^{1}$ and Christian Rylander ${ }^{4}$
}

\author{
* Correspondence: \\ Maria.Bergquist@gu.se \\ ${ }^{1}$ Department of Medical Sciences, \\ Clinical Physiology, Uppsala \\ University, S-75185 Uppsala, \\ Sweden \\ ${ }^{2}$ Department of Rheumatology and \\ Inflammation Research, Sahlgrenska \\ Academy, University of Gothenburg, \\ Gothenburg, Sweden \\ Full list of author information is \\ available at the end of the article
}

\begin{abstract}
Background: Glucocorticoid (GC) treatment has variable effect in sepsis. This may be explained by decreased expression or function of the glucocorticoid receptor (GR).

The aim of this study was to determine GR expression and binding capacity in patients during and after sepsis.
\end{abstract}

Methods: In this prospective, non-interventional clinical study, peripheral blood and clinical data were collected from 20 adult patients at five timepoints during sepsis and 5-13 months after recovery. GR expression and binding capacity were assessed by flow cytometry.

Results: GR expression was higher in T lymphocytes from patients with septic shock compared to healthy subjects $(p=0.01)$. While there was no difference in $G R$ expression between GC-treated and non-treated patients, GR binding capacity was lower in GC-treated patients at admission compared to healthy subjects ( $p \leq 0.03)$. After the acute inflammation inflammatory phase, GR binding capacity was still lower in neutrophils of GC-treated patients, compared to healthy subjects $(p=0.01)$. On admission, GR binding capacity in $T$ lymphocytes and neutrophils was inversely correlated with noradrenaline dose and lactate $(p \leq 0.03)$.

Conclusions: Our data suggest that GR expression is increased in T lymphocytes during septic shock regardless of GC treatment, while GR binding capacity is decreased in neutrophils in GC-treated patients. As neutrophils are the predominant circulating leucocyte in septic shock, their decreased GR binding capacity may impede the response to exogenous or endogenous glucocorticoids.

Keywords: Septic shock; Glucocorticoid receptor; Dexamethasone; Hydrocortisone; Cortisol; Human

\section{Background}

Steroid treatment for septic shock remains controversial due to contradictory results from clinical trials [1]. The main effects of endogenous and exogenous glucocorticoids (GC) are exerted through their binding to the intracellular glucocorticoid receptor (GR) present in all cells [2]. During early human sepsis, cortisol concentrations gradually rise with higher levels being inversely related to the chance of survival [3]. It is generally inferred that increased circulating cortisol during critical illness is a consequence of increased HPA axis activity and increased levels of ACTH, although ACTH levels have been found at similar levels or even below those of healthy controls [4-6].

\section{Springer}

C 2015 Bergquist et al. This is an Open Access article distributed under the terms of the Creative Commons Attribution License (http://creativecommons.org/licenses/by/4.0), which permits unrestricted use, distribution, and reproduction in any medium, provided the original work is properly credited. 
These findings suggest that rather than an increased production of cortisol, elevated cortisol levels are a consequence of a dysfunctional cortisol clearance from circulation which results in the supranormal cortisol levels found in the critically ill patients. Indeed, the expression and activity of A-ring reductases (the principal route of cortisol breakdown in humans) and $11 \beta$-HSD type 2 (converting cortisol to cortisone, inert to cells) were found reduced in the liver but not in the adipose tissue [6]. This raises the question whether the glucocorticoid receptor also has a decreased expression or function during critical illness. The importance of GR was recently illustrated by increased mortality, hemodynamic instability, and pro-inflammatory cytokine production found in mice, specifically lacking endothelial GR, subjected to endotoxic shock [7]. Our group recently showed that GR binding capacity was decreased in neutrophils during endotoxic shock in mice [8]. Previous quantitative studies of GR in critical illness investigated mRNA expression levels $[9,10]$, which precludes conclusions about the protein expression and subsequent receptor function. To the best of our knowledge, the effect of human sepsis on GR protein expression and GR binding capacity has not been investigated previously. The aim of this explorative, observational study was thus to describe GR expression and binding capacity in circulating $T$ lymphocytes and neutrophils, sampled from peripheral blood of adults during septic shock and after recovery.

\section{Methods}

\section{Patients and methods}

This multicenter study was approved by the Ethical Committee for Human Research in Uppsala, Sweden.

\section{Patients and healthy subjects}

Twenty patients were recruited between February 2012 and May 2013 in the general intensive care units (ICU) of the Sahlgrenska University Hospital and the Kungälv Hospital. During this period, 146 patients with septic shock were treated in the two ICUs, where hydrocortisone is used according to the Surviving Sepsis Campaign guidelines [11] at the discretion of the clinician. The selection of patients was influenced in a non-systematic way over time by the availability of laboratory resources. Inclusion criteria were (i) age over 18, (ii) sepsis according to the American-European consensus criteria [12], and (iii) treatment with at least one vasopressor. Exclusion criteria were (i) known infection with human immunodeficiency virus or hepatitis B or C, (ii) chronic treatment with GCs, (iii) participation in any investigational drug study within 4 weeks preceding the study period, and (iv) survival expected to be shorter than 3 days. Informed consent was initially obtained via next of kin and later directly from survivors. Consenting healthy subjects were recruited among non-smoking laboratory and hospital staff without any chronic or acute illness and with no medication.

Blood and clinical data were collected three times during septic shock, at T0 within $24 \mathrm{~h}$ of ICU admission, T1 $24 \mathrm{~h}$ after T0, and T2 48-120 h after T0 depending on logistics. Then, the blood was sampled when the acute inflammatory phase was considered to be over, and the patient was stable without support to vital functions at T3, 4-19 days after ICU admission. Finally, the blood was sampled after recovery at T4, 5-13 months after the patient had been discharged from 
the ICU. Survival was defined as alive 180 days after ICU admission. GR data from one patient at T0-T2 were lost due to technical reasons; one patient was lost to sampling at T3 and one to follow up at T4. One patient was excluded from sampling at T4 due to chronic GC treatment at that time. Clinical variables relevant for the degree of septic shock and organ dysfunction (blood pressure, heart rate, lactate, $\mathrm{ScvO}_{2}$, temperature, a-pH, base excess, serum creatinine, serum bilirubin, Glasgow Coma Scale (GCS) score, Simplified Acute Physiology Score (SAPS) III score, type and dose of vasoactive/inotropic agent) were registered for all timepoints where applicable. Blood gas values and clinical laboratory data (CRP, leucocyte and platelet counts, and microbiological cultures) were obtained from laboratory records. For plasma and serum, blood samples were centrifuged at $1500 \mathrm{~g}$ for $15 \mathrm{~min}$ and plasma was stored in $-70{ }^{\circ} \mathrm{C}$ until analysis.

\section{Cell preparation}

Whole blood aliquots were centrifuged with phosphate-buffered saline (PBS) at $1500 \mathrm{~g}$ and $4{ }^{\circ} \mathrm{C}$ for $10 \mathrm{~min}$ and subjected to erythrocyte lysis using BD FACS Lysing Solution (BD Biosciences, San José, CA, USA) for $15 \mathrm{~min}$ at room temperature. Leucocytes were then washed again twice with PBS and kept on ice until the total number of leucocytes was determined using an automated cell counter (Sysmex KX-21N, Kobe, Japan). For flow cytometry, a total of $5 \times 10^{5}$ cells were added to each well on a polypropylene conical 96-well plate (Thermo Fisher Scientific, Rochester, NY, USA) and stained with fluorochrome-conjugated antibodies binding to cell surface markers after Fc-blockage (Beriglobin ${ }^{\circ}$ CSL Behring, Denmark), anti-CD4 v450 (RPA-T4, BD Horizon ${ }^{\mathrm{ma}}$, BD Biosciences), anti-CD19 PerCP (4G7), anti-CD3 PerCP (SK7, BD Biosciences), anti-CD56 PE (B159), anti-CD16 Alexa Fluor 647 (3G8), anti-CD8 APC (RPA-T8), and anti-CD14 PE (M5E2 BD Pharmingen ${ }^{\mathrm{Tx}}$ ). Surface staining was performed at $+4{ }^{\circ} \mathrm{C}$ in the dark for $20 \mathrm{~min}$, followed by washing twice in FACS buffer (PBS supplemented with $100 \mathrm{mM}$ EDTA, $1 \%$ fetal calf serum, and $0.1 \%$ NaN3).

Cells were fixed and permeabilized for intracellular staining using a Fixation/ Permeabilization kit (eBioscience, San Diego, CA, USA) at $+4{ }^{\circ} \mathrm{C}$ for $30 \mathrm{~min}$. After washing twice in permeabilization buffer, Fc-blockage was performed in permeabilization buffer at $+4{ }^{\circ} \mathrm{C}$ in the dark for $10 \mathrm{~min}$. GR was labeled using anti-GR mAb (5E4, AbD Serotec, Dusseldorf, Germany) in permeabilization buffer at $+4{ }^{\circ} \mathrm{C}$ in the dark for $30 \mathrm{~min}$, followed by two washes with permeabilization buffer. Fluorescein isothiocyanate (FITC)-labeled mouse IgG1 isotype control $\left(\right.$ G18-145, BD Pharmingen $\left.{ }^{\mathrm{mm}}\right)$ was used as a negative control. For analysis, the cell pellets were resuspended in FACS buffer.

A separate set of samples for analysis of GR binding capacity was surface stained as described above but not permeabilized. Instead, cell pellets were resuspended in $10 \mu \mathrm{L}$ FACS buffer containing 20 nM FITC-conjugated dexamethasone (FITC-Dex) (Molecular Probes, Invitrogen, Carlsbad, CA, USA) [13]. Negative control samples were resuspended in $10 \mu \mathrm{L}$ FACS buffer containing $20 \mathrm{nM}$ dexamethasone (Merck, Darmstadt, Germany). After incubation at $+37^{\circ} \mathrm{C}$ in the dark for 30 min, cells were washed twice in FACS buffer and fixed with $4 \%$ paraformaldehyde at room temperature for $15 \mathrm{~min}$, then resuspended in FACS buffer and analyzed by flow cytometry. In an initial competitive experiment to exclude non-specific binding by FITC-Dex, cells were incubated with non-conjugated 
dexamethasone first, followed by a second incubation with FITC-Dex, which revealed a non-specific binding of less than $7 \%$ (data not shown).

\section{GR analysis}

GR expression and binding capacity in different leucocyte subsets were assessed using a FACS CantoII (BD Biosciences) equipped with Diva software (BD Biosciences). For each sample, at least 10,000 events were collected in the lymphocyte gate. Fluorescence was compensated using BD Anti-mouse CompBeads (BD Biosciences). For control of day-to-day variations of the assay, leucocytes from one healthy blood donor were included in each analysis. This internal standard was prepared sterile at one time as follows. Peripheral blood mononuclear cells (PBMCs) were separated using Lymphoprep (Fresenius Kabi, Oslo, Norway), washed in ice cold sterile PBS, and frozen in heat inactivated fetal calf serum with $15 \%$ DMSO at a rate of $-1{ }^{\circ} \mathrm{C} / \mathrm{min}$ using a Nalgene ${ }^{\circ} \mathrm{Mr}$. Frosty $^{\text {tm }}$ Freezing Container (Thermo Scientific, Waltham, MA, USA). For each analysis, a vial of the internal standard was defrosted on ice and washed in FACS buffer followed by surface and intracellular staining together with each patient sample. Data was further processed using FlowJo software (Tree Star, Inc., Ashland, VA, USA).

\section{Cytokine analysis}

Cytokines, granulocyte colony-stimulating factor (G-CSF), intracellular adhesion molecule 1 (ICAM-1), interferon alpha (IFN- $\alpha$ ), interferon gamma (IFN- $\gamma$ ), interleukin 1 alpha (IL-1 $\alpha$ ), interleukins (IL-1 $\beta$, IL-4, IL-6, IL-8, IL-10, IL-12p70, IL-13, IL-17A), interferon gamma-induced protein 10 (IP-10), latency-associated peptide (LAP), monocyte chemotactic protein 1 (MCP-1), macrophage inflammatory protein 1 alpha and beta (MIP-1 $\alpha$, MIP-1 $\beta$ ), tumor necrosis factor (TNF), and E-selectin, were analyzed in plasma using the Human Inflammation 20-plex RTU FlowCytomix Kit (eBioscience) according to manufacturer's recommendations. In short, $25 \mu \mathrm{L}$ of plasma was incubated with the bead mixture and biotin-conjugate at room temperature for $2 \mathrm{~h}$, shaking at $500 \mathrm{rpm}$. After wash, the samples were incubated with $50 \mu \mathrm{L}$ Streptavidin-PE solution at room temperature for $1 \mathrm{~h}$, shaking at $500 \mathrm{rpm}$. After final washes, the samples were analyzed in $280 \mu \mathrm{L}$ assay buffer using a FACS CantoII (BD Biosciences).

\section{Cortisol analysis}

For quantification of cortisol, plasma was analyzed using a commercially available EIA kit (Detect $\mathrm{X}^{\bullet}$ Cortisol, Arbor Assays, MI, USA), according to manufacturer's instructions. Optical density was read at $450 \mathrm{~nm}$ using a Spectra Max 340PC (Molecular Devices, Sunnyvale, CA, USA) running the SoftMax Pro 5.2 software.

\section{Statistical analysis}

Results were graphically presented with individual plots as well as group geometric means. Grouping of patients into GC-treated and GC-non-treated was based on GC treatment having been instituted or not at the time when samples were taken. Differences between groups were tested with the two-tailed Mann-Whitney test (GaphPad Prism 6.0 for Windows, GraphPad Software Inc, La Jolla, CA, USA). Associations 
between clinical parameters and GR expression or binding capacity at T0 in T lymphocytes and neutrophils were evaluated using the Spearman correlation test. The analyses were done using the $\mathrm{R}$ environment for statistical computing (version 3.1) [14] and the coin add-on package (version 1.1) [15]. $p$ values $\leq 0.05$ were considered statistically significant. Given the explorative nature of the study, multiple comparisons were not corrected for.

\section{Results}

\section{Patient and healthy subject characteristics}

Characteristics of the patients and the healthy subjects are listed in Table 1. Clinical parameters related to severity of shock are listed in Table 2. Fourteen of the 20 patients were treated with GC at some point, and six patients did not receive GC at any time. Two patients were GC-non-treated until T2 and T3, respectively. Of the clinical parameters, the plasma level of lactate and the dosage of noradrenaline were higher in the GC-treated than in the GC-non-treated patients at T0 $(p=0.004$ and 0.01 , respectively). Other shock-related parameters, however, did not differ between these groups. Out of $14 \mathrm{GC}$-treated and $6 \mathrm{GC}$-non-treated patients, 5 and 3 were dead after 180 days, respectively.

\section{Glucocorticoid receptor expression is elevated in T lymphocytes}

The GR expression in $\mathrm{T}$ lymphocytes and neutrophils showed a high intrainterindividual variation among patients as illustrated in Fig. 1a. Compared to healthy subjects, GR expression was higher in T lymphocytes $(p=0.01)$ but not in neutrophils of patients during the shock phase (T2). After recovery (T4), GR expression in T

Table 1 Demographics of patients and healthy subjects

\begin{tabular}{llll}
\hline Parameter & GC $(n=14)$ & No GC $(n=6)$ & Healthy subjects $(n=16)$ \\
\hline Men/women & $7 / 7^{\mathrm{a}}$ & $3 / 3$ & $3 / 12^{\mathrm{b}}$ \\
Age, mean (SD) years & $62.2(12.3)$ & $62.3(19.1)$ & $45.4(11.1)$ \\
Source of sepsis & & & \\
Lungs & 9 & 1 & \\
Abdomen & 2 & 2 & \\
Wound & 1 & 2 & \\
Urinary tract & 2 & 1 & \\
Unknown & 0 & 1 & \\
SAPS III, mean (SD) & $67.6(17.2)$ & $65.9(15.1)$ & \\
Alive/all at & & & \\
T0 & $12 / 12$ & $8 / 8$ & \\
T1 & $12 / 12$ & $8 / 8$ & \\
T2 & $13 / 13$ & $6 / 6$ & \\
T3 & $12 / 14$ & $3 / 6$ & \\
T4 & $9 / 14$ & & \\
\hline
\end{tabular}

GC patients treated with glucocorticoids, No GC patients not treated with glucocorticoids at any time

${ }^{\mathrm{a}} \mathrm{T}$ wo patients were GC-non-treated until T2 and T3, respectively

${ }^{b}$ Gender and age were not registered from one healthy subject

${ }^{\mathrm{c}}$ One patient had sepsis from two suspected sources 
Table 2 Clinical data related to shock

\begin{tabular}{|c|c|c|c|c|c|c|}
\hline \multirow[t]{2}{*}{ Parameter } & \multicolumn{3}{|l|}{ GC } & \multicolumn{3}{|l|}{ No GC } \\
\hline & TO & $\mathrm{T1}$ & $\mathrm{T} 2$ & T0 & $\mathrm{T} 1$ & $\mathrm{~T} 2$ \\
\hline Noradrenaline dose $[\mu \mathrm{g} / \mathrm{kg} / \mathrm{min}]$ & $0.45(0.12,0.87)^{a}$ & $0.55(0,1.46)^{a}$ & $0.18(0,1.1)$ & $0.15(0.09,0.25)$ & $0.10(0,0.32)$ & $0.05(0,0.23)$ \\
\hline Mean blood pressure $[\mathrm{mmHg}]$ & $71(60,88)$ & $68(55,90)$ & $83(66,112)$ & $69(34,90)$ & $75(62,90)$ & $86(70,100)$ \\
\hline Heart rate $[b p m]$ & $105(75,138)$ & $106(35,125)$ & $92(69,120)$ & $92(66,146)$ & $96(55,140)$ & $78(65,96)$ \\
\hline $\mathrm{ScvO}_{2}[\%]$ & $74(57,89)$ & $73(48,89)$ & $76(47,91)$ & $79(69,85)$ & $75(70,87)$ & $72(63,76)$ \\
\hline Temperature $\left[{ }^{\circ} \mathrm{C}\right]$ & $37.3(35.4,38.3)$ & $37.6(36.3,38.8)$ & $37.0(35.7,38.7)$ & $37.3(33.0,38.9)$ & $36.5(33.3,38.4)$ & $36.6(34.9,37.9)$ \\
\hline $\mathrm{a}-\mathrm{pH}$ & $7.29(7.18,7.43)$ & $7.31(7.13,7.49)$ & $7.34(7.22,7.45)$ & $7.35(7.26,7.42)$ & $7.35(7.26,7.47)$ & $7.37(7.30,7.41)$ \\
\hline Base excess & $-5.8(-10,1.3)$ & $-3.9(-15,4.3)$ & $0.4(-5.4,8.0)$ & $-3.1(-6.8,0.8)$ & $-1.6(-10,6.6)$ & $0.2(-2.8,3.4)$ \\
\hline Lactate & $5.5(1.3,14)^{\mathrm{a}}$ & $3.3(1.1,8.6)$ & $1.8(0.8,3.8)$ & $2.3(1.3,3.3)$ & $1.9(1.0,3.2)$ & $1.3(0.6,2.2)$ \\
\hline LPK & $8.3(1.3,30)$ & $11(1.3,23)$ & $12(1.5,22)$ & $14(1.3,28)$ & $11(3.3,24)$ & $9.4(3.9,18)$ \\
\hline TPK & $192(52,366)$ & $125(34,277)$ & $67(9.4,148)$ & $192(29,473)$ & $157(28,343)$ & $133(30,239)$ \\
\hline CRP & $167(49,290)$ & $215(52,410)$ & $154(46,330)$ & $213(140,336)$ & $247(92,328)$ & $151(46,315)$ \\
\hline
\end{tabular}

Numbers are given as median (range)

Difference between patients treated with glucocorticoids (GC) and patients not treated with glucocorticoids (No GC), $p<0.05$ 


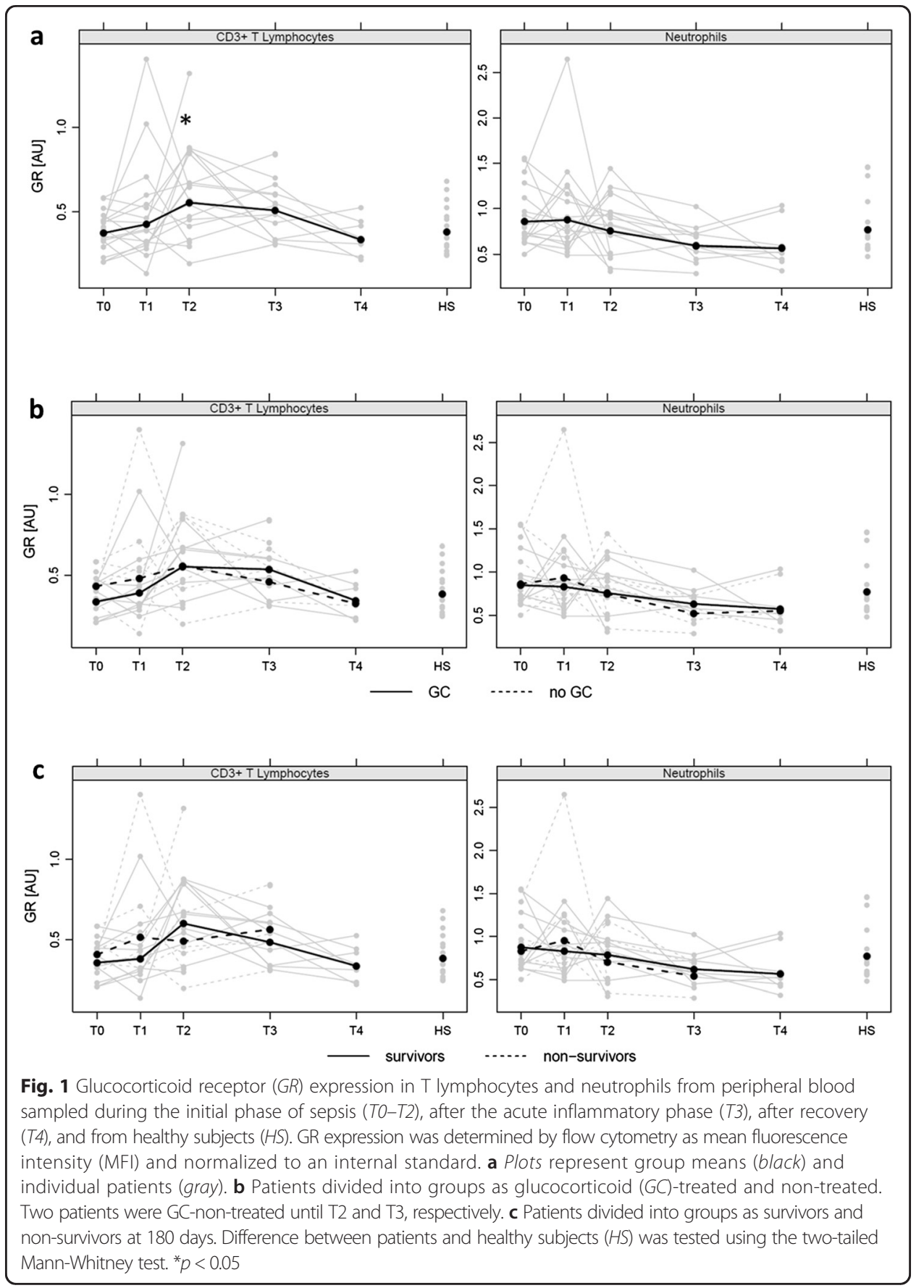

lymphocytes had retroceded to the level seen in healthy subjects. There was no difference in GR expression between GC-treated and GC-non-treated patients or between survivors and non-survivors at the different timepoints (Fig. 1b, c). However, the relative increase during septic shock (T0-T2) was higher in CD4+ T lymphocytes of survivors compared to non-survivors $(p=0.03)$. At T0, GR expression in T lymphocytes did not correlate with any of the clinical parameters, but in neutrophils, it was inversely correlated with a-pH $(p=0.03)$ and base excess $(p=0.02)$. 


\section{Glucocorticoid receptor binding capacity is reduced in neutrophils}

The GR binding capacity in all patients is illustrated in Fig. 2a. During septic shock, it did not differ from the GR binding capacity found in healthy subjects. However, when the acute inflammation had ceased (T3), GR binding capacity was lower in neutrophils of GC-treated patients compared to healthy subjects $(p=0.01)$. At the first measurement after admission (T0), GR binding capacity was lower in T lymphocytes $(p=0.008)$ and neutrophils $(p=0.03)$ of GC-treated patients compared to GC-non-treated patients (Fig. 2b). Non-survivors and survivors differed $(p=0.04)$ in evolution of GR binding
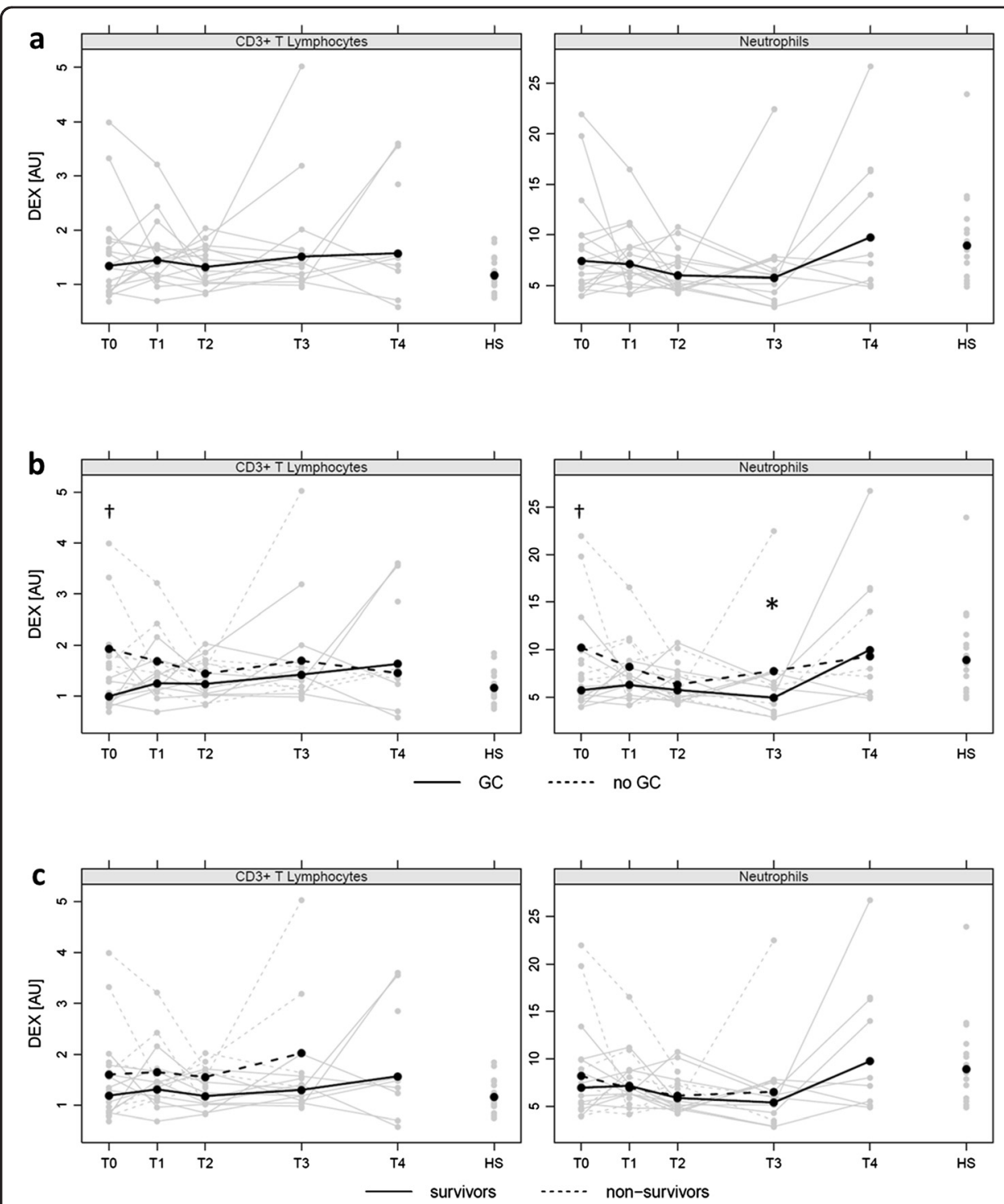

Fig. 2 Glucocorticoid receptor (GR) binding capacity in T lymphocytes and neutrophils from peripheral blood sampled during the initial phase of sepsis (T0-T2), after the acute inflammatory phase (T3), after recovery (T4), and from healthy subjects (HS). GR binding capacity (DEX) was determined by flow cytometry as mean fluorescence intensity (MFI) of fluorescence-labeled dexamethasone and normalized to an internal standard. a Plots represent group means (black) and individual patients (gray). b Patients divided into groups as glucocorticoid (GC)-treated and non-treated. Two patients were GC-non-treated until T2 and T3, respectively. c. Patients divided into groups as survivors and non-survivors at 180 days. Difference between patients and healthy subjects (HS) was tested using the two-tailed Mann-Whitney test. ${ }^{*} p<0.05$. Difference between GC-treated and non-treated patients was tested using the two-tailed Mann-Whitney test. ${ }^{\dagger} p<0.01$ 
capacity in neutrophils as non-survivors showed a relative increase while survivors showed a relative decrease between septic shock (T0) and their stabilized condition (T3). At T0, GR binding capacity in T lymphocytes was inversely correlated with IL-6, noradrenaline dose, and lactate $(p \leq 0.02)$, and in neutrophils, it was inversely correlated with noradrenaline dose and lactate $(p \leq 0.03)$ (Fig. 3).

\section{T lymphocyte numbers remain low after recovery}

The numbers of leucocytes on admission (T0) and after sepsis recovery (T4) are presented in Table 3. T lymphocytes were decreased, and neutrophils were increased after admission in septic shock (T0). After recovery (T4), patients treated with GC during sepsis still showed lower numbers of $\mathrm{T}$ and $\mathrm{B}$ lymphocytes compared to healthy subjects while neutrophil numbers were similar to the healthy subjects in both groups.

\section{Cortisol and cytokine response}

Shortly after ICU admission (T0), there were high levels of cortisol present in GCtreated patients as compared to healthy subjects $(p=0.01)$, reflecting both endogenous cortisol and hydrocortisone (Fig. 4). Most of the 20 cytokines analyzed displayed the highest concentration at T0, followed by a decline to levels similar to those of healthy subjects (Fig. 5). There were no significant differences between GC-treated and GCnon-treated patients although IL-6, IL-8, MCP-1, G-CSF, IL-17A, and IL-10 appeared higher in GC-treated patients, whereas TNF and IL-12p70 appeared higher in GC-nontreated patients at $\mathrm{T} 0$.

\section{Discussion}

The main findings of this study are that GR expression is upregulated in T lymphocytes during septic shock regardless of steroid treatment and that GR binding capacity is

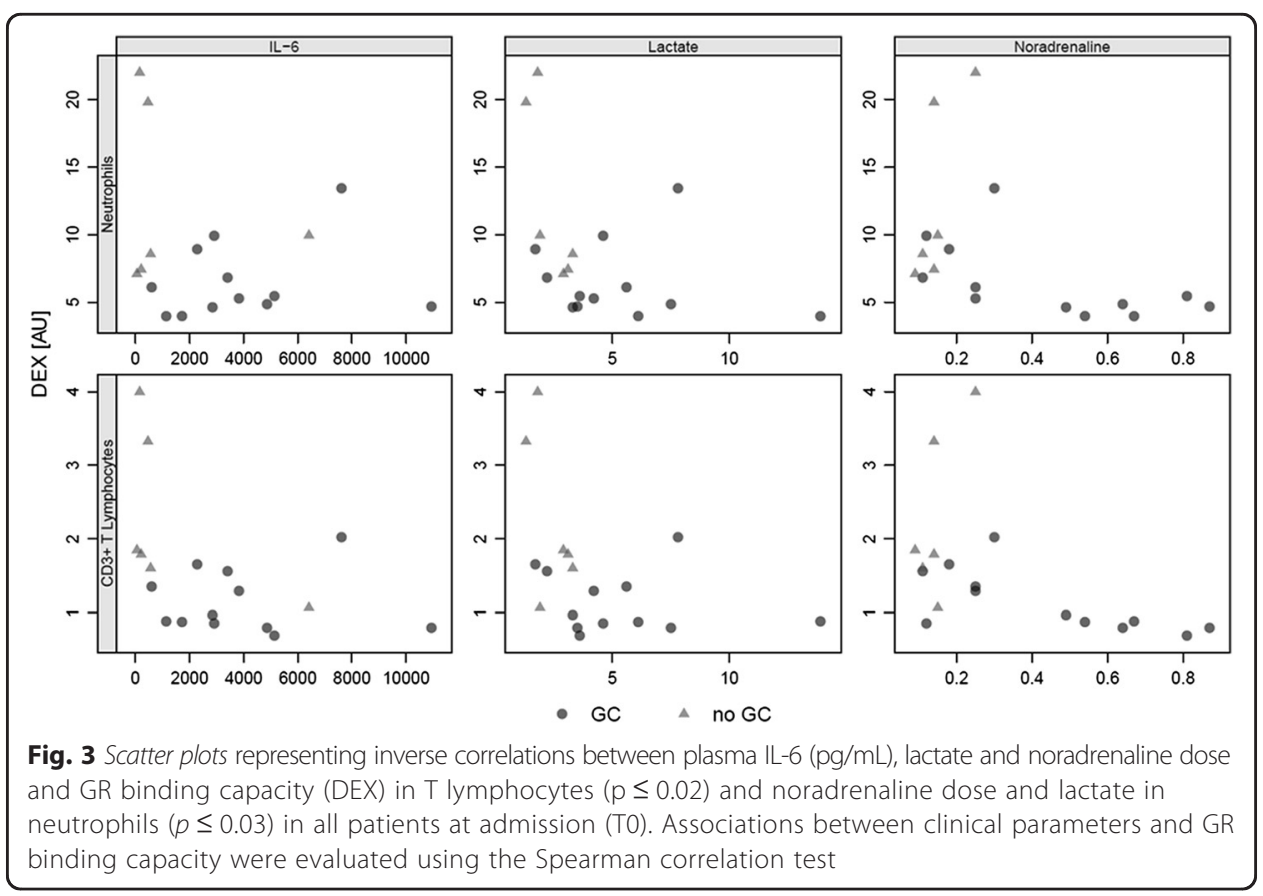


Table 3 Numbers of leucocytes per $\mathrm{mL}$ of peripheral blood

\begin{tabular}{|c|c|c|c|c|c|}
\hline & \multicolumn{4}{|l|}{ Patients } & \multirow[t]{3}{*}{ Healthy subjects } \\
\hline & \multicolumn{2}{|c|}{ On admission (T0) } & \multicolumn{2}{|c|}{ After recovery (T4) } & \\
\hline & No GC & GC & No GC & GC & \\
\hline CD3+ T lymphocytes & $2.3(2.6)^{* * *}$ & $3.6(4.9)^{* * *}$ & $11(4.6)$ & $9.4(6.7)^{*}$ & $18(7.9)$ \\
\hline CD4+ T lymphocytes & $1.1(1.1)^{* * *}$ & $2.3(4.3)^{* * *}$ & $5.1(1.4)$ & $4.3(3.9)^{*}$ & $10(4.4)$ \\
\hline CD8+ T Lymphocytes & $1.0(1.1)^{* * *}$ & $1.3(1.8)^{* * *}$ & $4.1(2.7)$ & $4.4(5.6)$ & $6.5(3.2)$ \\
\hline B lymphocytes & $1.6(2.7)$ & $1.0(1.7)$ & $1.9(2.0)$ & $0.6(0.7)^{*}$ & $2.0(1.3)$ \\
\hline NK cells & $0.8(1.4)$ & $0.8(0.7)$ & $3.6(2.3)$ & $0.8(0.8)$ & $2.1(2.0)$ \\
\hline Monocytes & $5.7(3.5)$ & $1.9(1.8)^{* * * a}$ & $3.0(2.1)$ & $3.8(2.5)$ & $5.3(2.5)$ \\
\hline Neutrophils & $145(63)^{* * *}$ & $76(66)^{a}$ & $28(8.3)$ & $41(29)$ & $51(23)$ \\
\hline Eosinophils & $0.9(1.2)$ & $0.8(2.2)^{*}$ & $1.8(0.4)$ & $2.0(1.4)$ & $2.9(2.6)$ \\
\hline
\end{tabular}

Numbers are $\times 10^{5}$, given as mean (SD)

${ }^{a}$ Difference between patients treated with glucocorticoids (GC) and patients not treated with glucocorticoids at any time (No GC), $p \leq 0.05$

${ }^{*}$ Difference between patients and healthy subjects (HS), $p \leq 0.05,{ }^{* *} p<0.001$

decreased in neutrophils after the inflammatory phase in patients who have been treated with steroids.

An upregulation of GR expression seems plausible as part of an adaptive response to dampen an aggressive inflammation in the presence of invading pathogens. Indeed, we previously have found increased GR protein expression in circulating leucocytes and splenocytes in experimental endotoxic shock in mice [8]. However, in earlier studies of GR mRNA, sepsis and septic shock were associated with decreased GR expression. According to Ledderose et al., using $\mathrm{T}$ lymphocytes from adult sepsis patients found a lower, however, not statistically significant, expression of GR- $\alpha$ than in healthy subjects [9]. van den Akker et al. studied neutrophils sampled from children within $24 \mathrm{~h}$ after admitted to a pediatric ICU for sepsis and septic shock and found that GR- $\alpha$ expression was slightly depressed but not statistically different from healthy subjects. However, GR expression increased with recovery to significantly higher levels than were found on admission [10].

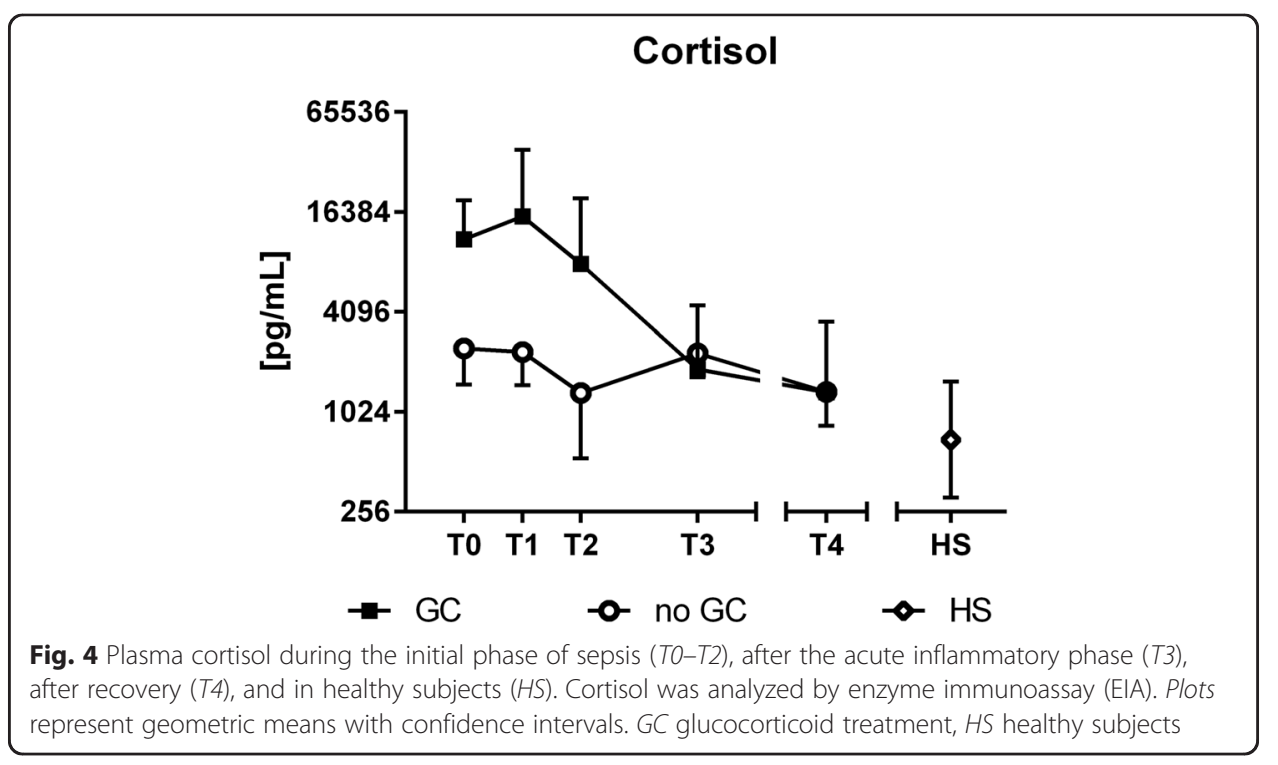




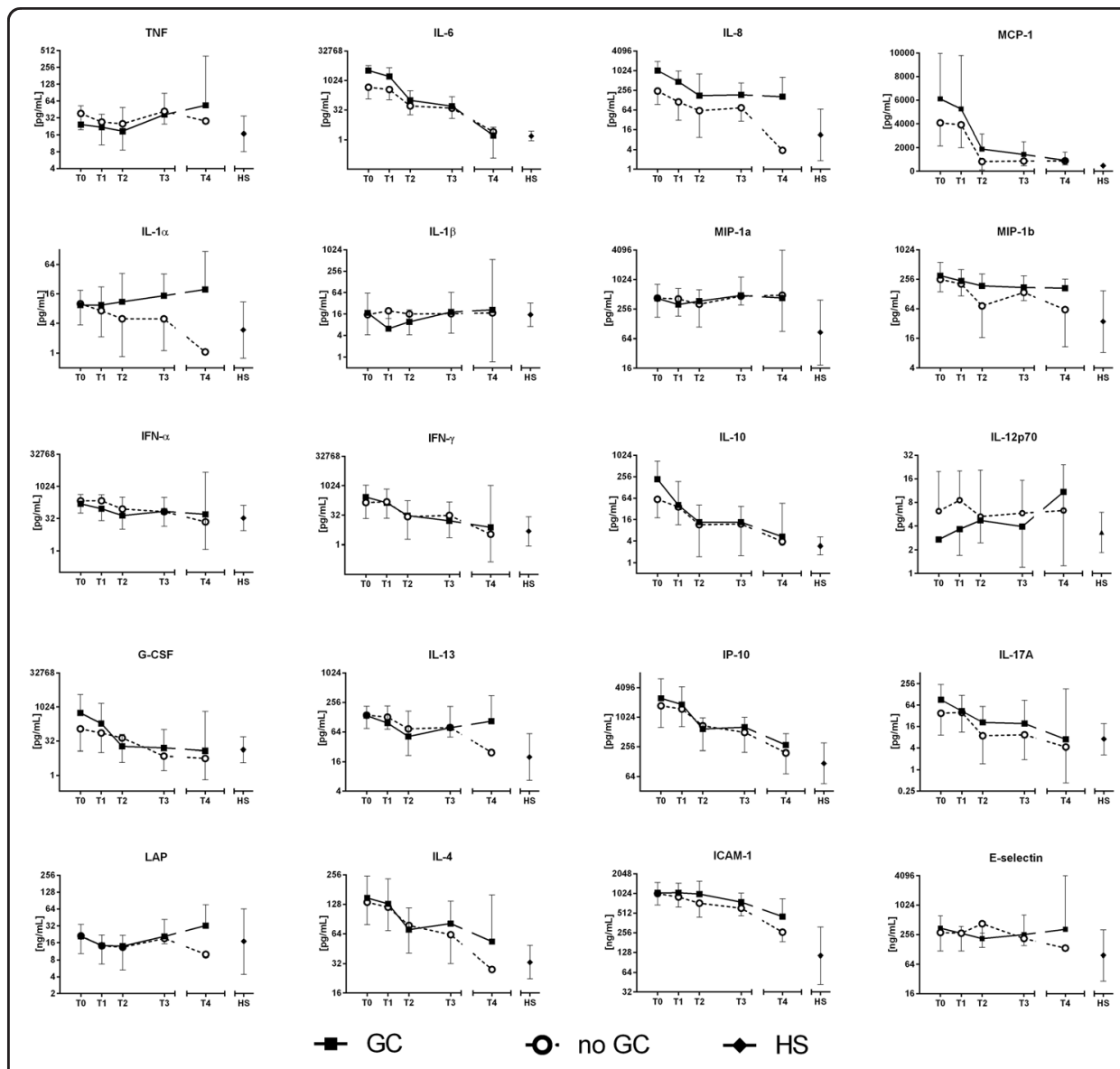

Fig. 5 Plasma cytokines during the initial phase of sepsis (T0-T2), after the acute inflammatory phase (T3), after recovery (T4), and in healthy subjects (HS). Cytokines were analyzed by flow cytometry. Plots represent geometric means with confidence intervals. GC glucocorticoid treatment, HS healthy subjects

These opposite findings may represent inconsistency between the GR protein and mRNA concentration as measures of GR expression. In an abnormal inflammatory situation such as sepsis, it is plausible that the speed of GR expression at the gene and protein levels can be restricted by a specific limiting factor. In eukaryotes, while mRNA can be produced in two copies per hour, dozens of the corresponding protein can be translated in the same time $[16,17]$. Transcription is estimated to be slower than translation because of several time-consuming steps, such as transcription initiation, intron excision, and posttranscriptional RNA processing, which are not required for protein translation. In addition, proteins have longer half-life than mRNAs (46 $\mathrm{h}$ versus $2.6-7 \mathrm{~h}$, respectively) [17].

Furthermore, we observed that survivors had a relatively higher increase of GR expression in T lymphocytes during septic shock than non-survivors. This clinical finding is supported by experimental results from Goodwin et al. who found that GR knockdown in endothelial cells proved to increase mortality and hemodynamic instability in mice subjected to endotoxic shock [7]. The importance of GR expression for survival of septic shock has been further demonstrated by Kleiman et al. who showed that GR dimerization-deficient mice were highly susceptible to endotoxic shock, suggestively by their inability to downregulate the pro-inflammatory cytokine IL-1 $\beta$ [18]. With our 
method, we did not investigate GR dimerization in itself but a high GR expression, as found in survivors, is a prerequisite for sufficient dimerization and function of the receptor.

The other, and perhaps most important finding in this study, was that GR binding capacity was decreased in neutrophils of patients treated with steroids. These patients were administered $200 \mathrm{mg}$ of hydrocortisone daily in line with current guidelines [11]. Their GR binding capacity was significantly lower than in healthy subjects at admission, but also, after septic shock was resolved. Impaired GR function has been described previously, in peripheral blood mononuclear cells in sepsis [19], lung tissue in experimental acute lung injury [20], and by our group in neutrophils in experimental endotoxic shock [8]. One possible interpretation would be that the GC-treated patients were in a deeper state of shock than the non-treated patients, causing decreased GR binding capacity. However, this explanation is unlikely as the patients from both groups were similar in most shock-related parameters and also because the difference between GCtreated and non-treated patients still remained at T3, when the shock was resolved. With the recent observation of substantial reduction in cortisol metabolism during critical illness [6], it is more likely that an excessively high concentration of endogenous cortisol and administered hydrocortisone caused decreased GR binding capacity. As the effects of signaling from the activated GR-GC complex during severe inflammation includes decreased capillary permeability, dampened cytokine release, and modulated leucocyte recruitment [21, 22], impaired GR binding capacity may have negative short-term consequences. Considering decreased binding capacity as well as the expanding number of neutrophils in sepsis, the inability of neutrophils to respond to endogenous or exogenous glucocorticoids may have severe ramifications for the host. In the presence of infection, while T lymphocytes display an increased GR expression, neutrophils may become resistant to glucocorticoid-induced apoptosis through decreased GR binding capacity. This is likely protecting the host from immunosuppression, which would exacerbate the present infection.

Our study also showed a severe retraction in the number of circulating $\mathrm{T}$ lymphocytes during sepsis. This is in agreement with previous studies [23] and may be an effect of the increased predisposition of $\mathrm{T}$ lymphocytes to undergo apoptosis in sepsis [24]. Neutrophils, on the other hand, were significantly increased in numbers during sepsis. Other studies have suggested that neutrophils are less sensitive to glucocorticoids than other leucocytes [25, 26]. Indeed, GR binding capacity in neutrophils was decreased by sepsis in our study. Taken together, these data suggest that $\mathrm{T}$ lymphocytes are the main glucocorticoid-responsive leucocytes, but may not be able to transmit an adequate effect of endogenous or exogenous glucocorticoids during sepsis due to their reduced number. Moreover, neutrophil activation is the predominant source of tissue damage and the multiple organ dysfunction syndrome (MODS) in critically ill patients [27, 28]. The acquired decreased binding capacity of neutrophils may be detrimental in sepsis and may even be aggravated by the increased circulating levels of glucocorticoids.

Interestingly, several months after recovery, survivors who received hydrocortisone treatment during sepsis in our study still had reduced numbers of CD3+ and CD4+ T lymphocytes and B lymphocytes well after recovery. GC-treated patients also showed higher concentrations of some cytokines at the time of recovery compared to any 
earlier timepoint and to healthy subjects. This is, to our knowledge, a novel observation. No patient displayed any symptom or sign of inflammation when blood samples were drawn after recovery, and it is therefore unlikely that they harbored any latent infection. If a decrease in $\mathrm{T}$ and $\mathrm{B}$ lymphocytes after sepsis recovery can be confirmed, this may explain why having survived sepsis is a strong predictor of developing subsequent infections [29]. Possible reasons for these findings remain speculative, and further studies are needed to confirm and delineate possible long-term cellular effects of glucocorticoid treatment during sepsis.

To the best of our knowledge, the expression and binding capacity has not previously been assessed on the protein level in septic shock. The method used in this study was partly developed by our group and is robust, offering information about both the GR expression at the protein level as well as its functional binding capacity ex vivo. It does not reveal information about the GR- $\alpha$ and GR- $\beta$ isoforms. As the GR- $\alpha$ and GR- $\beta$ isoforms originate from alternative splicing of the same gene, the only accurate way of specifically quantifying the GR- $\beta$ isoform is using $\mathrm{qPCR}$, which precludes information about both protein expression and function. For the present study, we chose to detect total GR expression and function using an antibody and fluorescence-labeled dexamethasone, respectively. The decreased GR binding capacity, that we observed using fluorescence-labeled dexamethasone, is unlikely to be an effect of competitive binding of hydrocortisone ex vivo. Naturally, occurring glucocorticoids (cortisol and hydrocortisone) dissociate within $15 \mathrm{~min}$ from GR, and any remaining unbound fraction would be washed away in the laboratory process. In addition, synthetic glucocorticoids have higher affinity for the receptor [30].

One limitation of the study is the non-consecutive inclusion (depending on available laboratory resources) of a relatively small study population, especially at the last timepoint (T4), and the demographic differences between patients and healthy subjects. The mean age of the healthy subjects was significantly lower, and immunological competence is known to decrease with age [31]. In addition, there were more women than men among the healthy subjects. However, when dividing the healthy subjects into two groups according to age or gender, we found no differences in GR expression or binding capacity. This does not preclude that age or gender had effects on the immune response in the patients, but they did not seem to affect our primary measured parameters in the healthy subjects. As this was an exploratory study, we did not make any multiplicity adjustments. Therefore, $p$ values should be interpreted with caution due to the increased risk for false positives. To the best of our knowledge, the expression and binding capacity has not previously been shown in septic shock. We consider the observations in this exploratory hypothesis-generating study interesting enough to merit validation in a larger hypothesis driven study, testing the correlation between GR binding capacity and outcome.

\section{Conclusions}

In summary, our data suggest that GR expression is increased in T lymphocytes during septic shock regardless of GC treatment, while GR binding capacity is decreased in neutrophils in GC-treated patients. As neutrophils are the predominant circulating leucocyte in septic shock, their decreased GR binding capacity may have severe consequences for the response to exogenous or endogenous glucocorticoids. Further studies 
are needed to investigate possible long-term immune traces of glucocorticoid treatment during sepsis.

\section{Abbreviations}

DEX: dexamethasone; GC: glucocorticoid; GR: glucocorticoid receptor.

\section{Competing interests}

The authors declare that they have no competing interests.

\section{Authors' contributions}

MB contributed in the planning of the study, carried out the laboratory analyses, interpreted the data, and drafted the manuscript. CL participated in the method development and data interpretation. MS contributed in the study design and recruited patients. GH conceived of the study and participated in the data interpretation and manuscript drafting. CR contributed in the design of the study, recruited patients, participated in the data interpretation and manuscript drafting. All authors read and approved the manuscript

\section{Acknowledgements}

The authors are indebted to Doctor Jenny Skytte and Research Nurses Caisa Jacobsson and Anette Spain at the Sahlgrenska University Hospital. This work was supported by grant 5315 from the Swedish Research Council, by grant 308301 from the Regional Research Funds of Västra Götaland, and by grants from the Inga-Britt and Arne Lundberg Foundation.

This study is financially supported by the Swedish Research council no 5315 (GH) and Regional Research Fund of Västra Götaland no $308301(\mathrm{CR})$

\section{Author details}

${ }^{1}$ Department of Medical Sciences, Clinical Physiology, Uppsala University, S-75185 Uppsala, Sweden. ${ }^{2}$ Department of Rheumatology and Inflammation Research, Sahlgrenska Academy, University of Gothenburg, Gothenburg, Sweden. ${ }^{3}$ Department of Anesthesia and Intensive Care, Kungälv Hospital, Kungälv, Sweden. ${ }^{4}$ Department of Anesthesia and Intensive Care, Sahlgrenska University Hospital, Gothenburg, Sweden.

Received: 4 May 2015 Accepted: 13 July 2015

Published online: 28 July 2015

\section{References}

1. Patel GP, Balk RA (2012) Systemic steroids in severe sepsis and septic shock. Am J Respir Crit Care Med 185(2):133-139

2. Allary J, Annane D (2005) Glucocorticoids and sepsis. Minerva Anestesiol 71(12):759-768

3. Zhang Q, Dong G, Zhao X, Wang M, Li CS (2014) Prognostic significance of hypothalamic-pituitary-adrenal axis hormones in early sepsis: a study performed in the emergency department. Intensive Care Med 40(10):1499-1508

4. Vermes I, Beishuizen A, Hampsink RM, Haanen C (1995) Dissociation of plasma adrenocorticotropin and cortisol-levels in critically ill patients: possible role of endothelin and atrial natriuretic hormone. J Clin Endocrinol Metabol 80(4):1238-1242

5. Polito A, Sonneville R, Guidoux C, Barrett L, Viltart O, Mattot V, Siami S, de la Grandmaison GL, Chretien F, Singer M, Gray F, Annane D, Brouland J-P, Sharshar T (2011) Changes in CRH and ACTH synthesis during experimental and human septic shock. Plos One 6(11), e25905

6. Boonen E, Vervenne H, Meersseman P, Andrew R, Mortier L, Declercq PE, Vanwijngaerden Y-M, Spriet I, Wouters PJ, Vander Perre S, Langouche L, Vanhorebeek I, Walker BR, Van den Berghe G (2013) Reduced cortisol metabolism during critical illness. New Engl J Med 368(16):1477-1488

7. Goodwin JE, Feng Y, Velazquez H, Sessa WC (2013) Endothelial glucocorticoid receptor is required for protection against sepsis. Proc Natl Acad Sci U S A 110(1):306-311

8. Bergquist M, Jirholt P, Nurkkala M, Rylander C, Hedenstierna G, Lindholm C (2014) Glucocorticoid receptor function is decreased in neutrophils during endotoxic shock. J Infect 69(2):113-122

9. Ledderose C, Moehnle P, Limbeck E, Schuetz S, Weis F, Rink J, Briegel J, Kreth S (2012) Corticosteroid resistance in sepsis is influenced by microRNA-124-induced downregulation of glucocorticoid receptor-alpha. Crit Care Med 40(10):2745-2753

10. van den Akker ELT, Koper JW, Joosten K, de Jong FH, Hazelzet JA, Lamberts SWJ, Hokken-Koelega ACS (2009) Glucocorticoid receptor mRNA levels are selectively decreased in neutrophils of children with sepsis. Intensive Care Med 35(7):1247-1254

11. Dellinger RP, Levy MM, Rhodes A, Annane D, Gerlach H, Opal SM, Sevransky JE, Sprung CL, Douglas IS, Jaeschke R, Osborn TM, Nunnally ME, Townsend SR, Reinhart K, Kleinpell RM, Angus DC, Deutschman CS, Machado FR, Rubenfeld GD, Webb SA, Beale RJ, Vincent JL, Moreno R (2013) Surviving sepsis campaign: International Guidelines for Management of Severe Sepsis and Septic Shock: 2012. Crit Care Med 41(2):580-637

12. Levy MM, Fink MP, Marshall JC, Abraham E, Angus D, Cook D, Cohen J, Opal SM, Vincent JL, Ramsay G (2003) 2001 SCCM/ESICM/ACCP/ATS/SIS International Sepsis Definitions Conference. Crit Care Med 31(4):1250-1256

13. Du J, Li M, Zhang D, Zhu X, Zhang W, Gu W, Feng Y, Zhai X, Ling C (2009) Flow cytometry analysis of glucocorticoid receptor expression and binding in steroid-sensitive and steroid-resistant patients with systemic lupus erythematosus. Arthritis Res Ther 11(4):R108

14. Team RC (2014) R: a language and environment for statistical computing. R Foundation for Statistical Computing, Vienna, Austria

15. Hothorn T, Hornik K, van de Wiel MA, Zeileis A (2006) A lego system for conditional inference. Am Stat 60(3):257-263 
16. Schwanhausser B, Busse D, Li N, Dittmar G, Schuchhardt J, Wolf J, Chen W, Selbach M (2011) Global quantification of mammalian gene expression control. Nature 473(7347):337-342

17. Vogel C, Marcotte EM (2012) Insights into the regulation of protein abundance from proteomic and transcriptomic analyses. Nat Rev Genet 13(4):227-232

18. Kleiman A, Huebner S, Parkitna JMR, Neumann A, Hofer S, Weigand MA, Bauer M, Schmid W, Schuetz G, Libert C, Reichardt HM, Tuckermann JP (2012) Glucocorticoid receptor dimerization is required for survival in septic shock via suppression of interleukin-1 in macrophages. Faseb Journal 26(2):722-729

19. Molijn GJ, Koper JW, Vanuffelen CJC, Dejong FH, Brinkmann AO, Bruining HA, Lamberts SWJ (1995) Temperature-induced down-regulation of the glucocorticoid receptor in peripheral blood mononuclear leucocytes in patients with sepsis or septic shock. Clin Endocrinol 43(2):197-203

20. Liu LY, Sun B, Tian Y, Lu BZ, Wang J (1993) Changes of pulmonary glucocorticoid receptor and phospholipase A2 in sheep with acute lung injury after high-dose endotoxin infusion. Am Rev Respir Dis 148(4):878-881

21. Williams TJ, Yarwood H (1990) Effect of glucocorticosteroids on microvascular permeability. Am Rev Respir Dis 141(2):S39-S43

22. Skubitz KM, Craddock PR, Hammerschmidt DE, August JT (1981) Corticosteroids block binding of chemotactic peptide to its receptor on granulocytes and cause disaggregation of granulocyte aggregates in vitro. J Clin Investig 68(1):13-20

23. Hotchkiss RS, Tinsley KW, Swanson PE, Schmieg RE, Hui JJ, Chang KC, Osborne DF, Freeman BD, Cobb JP, Buchman TG, Karl IE (2001) Sepsis-induced apoptosis causes progressive profound depletion of B and CD4(+) T lymphocytes in humans. J Immunol 166(11):6952-6963

24. Hotchkiss RS, Swanson PE, Cobb JP, Jacobson A, Buchman TG, Karl IE (1997) Apoptosis in lymphoid and parenchymal cells during sepsis: Findings in normal and T- and B-cell-deficient mice. Crit Care Med 25(8):1298-1307

25. Schleimer RP (1990) Effects of glucocorticosteroids on inflammatory cells relevant to their therapeutic applications in asthma. Am Rev Respir Dis 141(2):S59-S69

26. Wen WP, Liu WL, Zhang L, Bai J, Fan YP, Xia WT, Luo Q, Zheng J, Wang HT, Li ZW, Xia JH, Jiang HY, Liu Z, Shi JB, $\mathrm{Li} \mathrm{HB}, \mathrm{Xu} \mathrm{G}(2012)$ Increased neutrophilia in nasal polyps reduces the response to oral corticosteroid therapy. J Allergy Clin Immunol 129(6):1522-1528

27. Thijs A, Thijs LG (1998) Pathogenesis of renal failure in sepsis. Kidney Int 53:S34-S37

28. Brealey D, Singer M (2000) Multi-organ dysfunction in the critically ill: effects on different organs. J R Coll Physicians Lond 34(5):428-431

29. Wang T, Derhovanessian A, De Cruz S, Belperio JA, Deng JC, Hoo GS (2014) Subsequent infections in survivors of sepsis: epidemiology and outcomes. J Intensive Care Med 29(2):87-95

30. Stavreva DA, Wiench M, John S, Conway-Campbell BL, McKenna MA, Pooley JR, Johnson TA, Voss TC, Lightman SL, Hager GL (2009) Ultradian hormone stimulation induces glucocorticoid receptor-mediated pulses of gene transcription. Nat Cell Biol 11(9):1093-1102

31. Inoue S, Suzuki-Utsunomiya K, Okada Y, lida Y, Taira T, Miura N, Tsuji T, Yamagiwa T, Morita S, Chiba T, Sato T, Inokuchi S (2013) Reduction of immunocompetent t cells followed by prolonged lymphopenia in severe sepsis in the elderly. Crit Care Med 41(3):810-819

\section{Submit your manuscript to a SpringerOpen ${ }^{\circ}$ journal and benefit from:}

- Convenient online submission

Rigorous peer review

- Immediate publication on acceptance

- Open access: articles freely available online

- High visibility within the field

- Retaining the copyright to your article 